\title{
Integration and Optimization of Multivariate Polynomials by Restriction onto a Random Subspace
}

\author{
Alexander Barvinok \\ Department of Mathematics \\ University of Michigan \\ Ann Arbor, MI 48109-1043, USA \\ barvinok@umich.edu
}

\begin{abstract}
We consider the problem of efficient integration of an $n$-variate polynomial with respect to the Gaussian measure in $\mathbb{R}^{n}$ and related problems of complex integration and optimization of a polynomial on the unit sphere. We identify a class of $n$-variate polynomials $f$ for which the integral of any positive integer power $f^{p}$ over the whole space is well approximated by a properly scaled integral over a random subspace of dimension $O(\log n)$. Consequently, the maximum of $f$ on the unit sphere is well approximated by a properly scaled maximum on the unit sphere in a random subspace of dimension $O(\log n)$. We discuss connections with problems of combinatorial counting and applications to efficient approximation of a hafnian of a positive matrix.
\end{abstract}

\section{Introduction}

We consider the problem of efficient integration of multivariate polynomials with respect to the Gaussian measure in $\mathbb{R}^{n}$.

Let us assume that a real $n$-variate homogeneous polynomial $f$ of degree $m$ is given to us by some "black box," which inputs an $n$-vector $x$ and outputs the value

Date received: March 21, 2005. Final version received: October 13, 2005. Date accepted: October 24, 2005. Communicated by Arieh Iserles. Online publication: March 6, 2006.

AMS classification: 68W20, 68W25, 60D05, 90C26.

Key words and phrases: Polynomials, Integration, Wick formula, Algorithms, Random subspaces, Gaussian measure. 
of $f(x)$. We want to compute or estimate the integral

$$
\int_{\mathbb{R}^{n}} f d \mu_{n}
$$

where $\mu_{n}$ is the standard Gaussian measure with the density

$(2 \pi)^{-n / 2} e^{-\|x\|^{2} / 2}, \quad$ where $\quad\|x\|=\left(\xi_{1}^{2}+\cdots+\xi_{n}^{2}\right)^{1 / 2} \quad$ for $\quad x=\left(\xi_{1}, \ldots, \xi_{n}\right)$.

If $m$ is odd, then the integral is 0 , so the interesting case is that of an even degree $m$.

An equivalent problem is to integrate $f$ over the unit sphere $\mathbb{S}^{n-1} \subset \mathbb{R}^{n}$. Since $f$ is homogeneous of degree $m$, passing to the polar coordinates we obtain

$$
\int_{\mathbb{R}^{n}} f d \mu_{n}=(2 \pi)^{-n / 2}\left(\int_{0}^{+\infty} r^{n-1+m} e^{-r^{2} / 2} d r\right)\left(\int_{\mathbb{S}^{n-1}} f(x) d x\right),
$$

from which for $m=2 k$ we get

$$
\int_{\mathbb{S}^{n-1}} f d \omega_{n}=\frac{\Gamma(n / 2)}{2^{k} \Gamma(n / 2+k)} \int_{\mathbb{R}^{n}} f d \mu_{n},
$$

where $\omega_{n}$ is the rotation invariant Haar probability measure on $\mathbb{S}^{n-1}$. This and related formulas for integrals of polynomials over the unit sphere and over the Gaussian measure on $\mathbb{R}^{n}$ can be found, for example, in [2].

The most straightforward approach to integration is to employ the Monte Carlo method, that is, to sample $N$ random points $x_{i} \in \mathbb{S}^{n-1}$ and approximate the integral by the sample mean:

$$
\int_{\mathbb{S}^{n-1}} f d \omega_{n} \approx \frac{1}{N} \sum_{i=1}^{N} f\left(x_{i}\right) .
$$

Although one can show that for a "typical" polynomial the Monte Carlo method works reasonably well, there are simple examples of polynomials $f$ where one would require to sample exponentially many points to approximate the integral within a reasonable relative error.

(1.1) Example. Suppose that $f(x)=\xi_{1}^{2 k}$ for $x=\left(\xi_{1}, \ldots, \xi_{n}\right)$. Then

$$
\int_{\mathbb{S}^{n-1}} f d \omega_{n}=\frac{\Gamma(n / 2) \Gamma(1 / 2+k)}{\sqrt{\pi} \Gamma(n / 2+k)} .
$$

Let $k=\lfloor n / 2\rfloor$. A straightforward consequence of Stirling's formula is that the integral is of the order of $2^{-n}$ for large $n$.

On the other hand, if we sample $N$ random points $x_{i}$ on the unit sphere $\mathbb{S}^{n-1}$, then with high probability we will have $\left|\xi_{1}\right|=O\left((\ln N)^{1 / 2} n^{-1 / 2}\right)$ for the first coordinate $\xi_{1}$ of every sampled point. Indeed, for a random point $x \in \mathbb{S}^{n-1}$ we have $\left|\xi_{1}\right|>\varepsilon$ 
with probability at most $\sqrt{\pi / 2} e^{-\varepsilon^{2}(n-2)}$, see, for example, Section 2 of [11], so a randomly sampled point $x \in \mathbb{S}^{n-1}$ hits the region $\left|\xi_{1}\right|>(\ln N)^{1 / 2}(n-2)^{-1 / 2}$ with probability $O\left(N^{-1}\right)$. Thus to approximate the integral within a factor of $10^{n}$, the number $N$ of samples should be exponentially large in $n$.

The reason why the Monte Carlo method doesn't work well on the above example is clear: the polynomial $f(x)=\xi_{1}^{2 k}$ acquires some large values for an exponentially small fraction of $x \in \mathbb{S}^{n-1}$ but those values significantly contribute to the integral. See also [3] for a discussion of the relevant "measure concentration phenomenon" within the general context of numerical analysis.

Thus the Monte Carlo method wouldn't work well if the graph of the polynomial looks "needle-like." In this paper, we suggest a method tailored specifically for such needle-like polynomials. The following defines the class of "needle-like" or "focused" polynomials we deal with.

(1.2) Definitions. Let

$$
\langle x, y\rangle=\xi_{1} \eta_{1}+\cdots+\xi_{n} \eta_{n} \quad \text { for } \quad x=\left(\xi_{1}, \ldots, \xi_{n}\right) \quad \text { and } \quad y=\left(\eta_{1}, \ldots, \eta_{n}\right)
$$

be the standard scalar product in $\mathbb{R}^{n}$.

Let us fix a number $0<\delta \leq 1$ and a positive integer $N$. We say that a homogeneous polynomial $f: \mathbb{R}^{n} \rightarrow \mathbb{R}$ of degree $m$ is $(\delta, N)$-focused if there exist $N$ nonzero vectors $c_{1}, \ldots, c_{N} \in \mathbb{R}^{n}$ such that:

- for every pair $(i, j)$ the cosine of the angle between $c_{i}$ and $c_{j}$ is at least $\delta$;

- the polynomial $f$ can be written as a nonnegative linear combination

$$
f(x)=\sum_{I} \alpha_{I} \prod_{i \in I}\left\langle c_{i}, x\right\rangle,
$$

where the sum is taken over subsets $I \subset\{1, \ldots, N\}$ of cardinality $|I|=m$ and $\alpha_{I} \geq 0$.

Here are some examples of $(\delta, N)$-focused polynomials.

\section{(1.3) Examples.}

(1.3.1) Let $c_{1}, \ldots, c_{N} \in \mathbb{R}^{n}$ be vectors with positive coordinates such that the ratio of the smallest/largest coordinate for each vector $c_{i}$ is at least $\sqrt{\delta}$. Then the cosine of the angle between $c_{i}$ and $c_{j}$ is at least $\delta$ for each pair $(i, j)$. Indeed, scaling $c_{1}, \ldots, c_{N}$, if necessary, we may assume that the largest coordinate of every $c_{i}$ is 1. Then $\left\langle c_{i}, c_{j}\right\rangle \geq n \delta$ while $\left\|c_{i}\right\|,\left\|c_{j}\right\| \leq \sqrt{n}$. Thus the polynomial $f$ defined by (1.2.1) will be $(\delta, N)$-focused. Bounding the ratio of the smallest/largest coordinate of $c_{i}$ can be considered as a strengthening of the condition that $f$ has nonnegative coefficients: if we allow $c_{i}$ in (1.2.1) to be arbitrary nonnegative vectors, we obtain the class of all polynomials $f$ with nonnegative coefficients. 
(1.3.2) Suppose that $n=k(k+1) / 2$ and let us identify $\mathbb{R}^{n}$ with the space of $k \times k$ symmetric matrices with the scalar product $\langle a, b\rangle=\operatorname{trace}(a b)$. Let $c_{1}, \ldots, c_{N}$ be positive definite matrices such that the ratio of the smallest/largest eigenvalue for each matrix $c_{i}$ is at least $\sqrt{\delta}$. As above, one can show that the cosine of the angle between $c_{i}$ and $c_{j}$ is at least $\delta$ for each pair $(i, j)$. Indeed, without loss of generality, we may assume that $c_{i}$ is diagonal and reduce the case to (1.3.1).

Other instances can be obtained by sampling $c_{1}, \ldots, c_{N}$ in (1.2.1) at random from some biased distribution in $\mathbb{R}^{n}$ (a distribution with a nonzero expectation). Further instances of $(\delta, N)$-focused polynomials can be obtained by taking expectations of such random polynomials and approximating them by sample averages. For example, the complete symmetric polynomial $h_{m}(x)$ of degree $m$ can be obtained as the expectation of $(m !)^{-1}\langle c, x\rangle^{m}$, where the coefficients of $c$ are sampled from the standard exponential distribution.

Our first result is that the value of the integral of a focused polynomial over a random lower-dimensional subspace allows one to predict the value of the integral over the whole space.

For a $k$-dimensional subspace $L \subset \mathbb{R}^{n}$, let $\mu_{k}$ be the Gaussian measure concentrated on $L$ with the density $(2 \pi)^{-k / 2} \exp \left\{-\|x\|^{2} / 2\right\}$ for $x \in L$. We pick a $k$-dimensional subspace at random with respect to the Haar probability measure on the Grassmannian $G_{k}\left(\mathbb{R}^{n}\right)$ and consider the integral

$$
\int_{L} f d \mu_{k}
$$

(see Section 1 of [11] for a succinct introduction to the Haar measure, including that on the Grassmannian).

We claim that as long as $k \sim \log N$, the properly scaled integral over $L$ approximates the integral over $\mathbb{R}^{n}$ within a factor of $(1-\varepsilon)^{m / 2}$.

(1.4) Theorem. There exists an absolute constant $\gamma>0$ with the following property.

For any $\delta>0$, for any positive integer $N$, for any $(\delta, N)$-focused polynomial $f: \mathbb{R}^{n} \rightarrow \mathbb{R}$ of degree $m$, for any $\varepsilon>0$, and any positive integer $k \geq \gamma \varepsilon^{-2} \delta^{-2} \ln (N+2)$, the inequality

$$
(1-\varepsilon)^{m / 2} \int_{L} f d \mu_{k} \leq\left(\frac{k}{n}\right)^{m / 2} \int_{\mathbb{R}^{n}} f d \mu_{n} \leq(1-\varepsilon)^{-m / 2} \int_{L} f d \mu_{k}
$$

holds with probability at least $2 / 3$ for a random $k$-dimensional subspace $L \subset \mathbb{R}^{n}$.

Assuming that we can integrate efficiently over lower-dimensional subspaces (see Section 1.6 below), we get a randomized approximation algorithm for computing the integral of $f$ over $\mathbb{R}^{n}$. Namely, we sample a random $k$-dimensional 
subspace $L$, compute the integral over $L$, and output the value of that integral multiplied by $(n / k)^{m / 2}$. To sample $L$ from the uniform distribution on the Grassmannian $G_{k}\left(\mathbb{R}^{n}\right)$, one can sample $k$ vectors $x_{1}, \ldots, x_{k}$ independently from the Gaussian distribution in $\mathbb{R}^{n}$ and let $L=\operatorname{span}\left\{x_{1}, \ldots, x_{k}\right\}$.

One "anti-Monte Carlo" feature of the algorithm is that the estimator is decidedly biased: the expected value of the output is essentially greater (by a factor of $\left.(n / k)^{m / 2}\right)$ than the value we are trying to approximate. This is so because the distribution of the integral over a random subspace has a "thick tail": there are subspaces which result in large integrals that significantly contribute to the integral over the whole space but such subspaces are very rare.

To increase the probability of obtaining the right approximation, one can use the standard approach of sampling several random subspaces and finding the median value of the outputs.

One can observe that if $f$ is $(\delta, N)$-focused, then $f^{p}$ is also $(\delta, N)$-focused for any positive integer $p$. This allows us to deduce that the maximum of $f$ over the unit sphere is well approximated by the scaled maximum of the restriction of $f$ onto the sphere in a lower-dimensional subspace.

(1.5) Corollary. There exists an absolute constant $\gamma>0$ with the following property.

For any $\delta>0$, for any positive integer $N$, for any $(\delta, N)$-focused polynomial $f: \mathbb{R}^{n} \rightarrow \mathbb{R}$ of degree $m$, for any $\varepsilon>0$, and any positive integer $k \geq$ $\gamma \varepsilon^{-2} \delta^{-2} \ln (N+2)$, the inequality

$$
(1-\varepsilon)^{m / 2} \max _{x \in \mathbb{S}^{n-1} \cap L} f(x) \leq\left(\frac{k}{n}\right)^{m / 2} \max _{x \in \mathbb{S}^{n-1}} f(x) \leq(1-\varepsilon)^{-m / 2} \max _{x \in \mathbb{S}^{n-1} \cap L} f(x)
$$

holds with probability at least $2 / 3$ for a random $k$-dimensional subspace $L \subset \mathbb{R}^{n}$.

The problem of optimization of a polynomial on the unit sphere has attracted some attention recently, see [5] and [9]. Note that by restricting the polynomial onto a $k$-dimensional subspace we effectively reduce the number of variables to $k$ in the optimization problem. Using methods of computational algebraic geometry allows one to optimize a polynomial over the sphere in time exponential in the number of variables [12]. Hence with $k=O(\log N)$, we obtain a quasi-polynomial algorithm of $m^{O(\log N)}$ complexity which approximates the maximum value of the polynomial on the sphere within a $(1-\varepsilon)^{m / 2}$ factor. If the degree $m$ of the polynomial is fixed and $N$ is bounded by a polynomial in the number $n$ of variables, we get a polynomial time approximation algorithm.

In particular, whenever we have a polynomial $f$ as in (1.2.1) and vectors $c_{i}$ as in (1.3.1)-(1.3.2), integration (optimization) of $f$ over the unit sphere $\mathbb{S}^{n-1}$ reduces to integration (optimization) of $f$ over a random lower-dimensional subspace $L$. 
(1.6) On the Computational Complexity. Let $f: \mathbb{R}^{n} \rightarrow \mathbb{R}$ be a homogeneous polynomial of degree $m$ given by its "black box" which outputs the value of $f(x)$ for an input $x \in \mathbb{R}^{n}$. Then one can compute the monomial expansion

$$
f(x)=\sum_{\alpha} c_{\alpha} \mathbf{x}^{\alpha} \quad \text { where } \quad \mathbf{x}^{\alpha}=x_{1}^{\alpha_{1}} \ldots x_{n}^{\alpha_{n}} \quad \text { for } \quad \alpha=\left(\alpha_{1}, \ldots, \alpha_{n}\right)
$$

in $O\left(\left(\begin{array}{c}n+m-1 \\ m\end{array}\right)^{3}\right)$ time through the standard procedure of interpolation, see also [8] for the sparse version. If $L \subset \mathbb{R}^{n}$ is a $k$-dimensional subspace, by choosing an orthonormal basis in $L$, we can identify $L$ with $\mathbb{R}^{k}$. Then the monomial expansion of the restriction $f_{L}$ can be computed in $\left.O\left(\left(\begin{array}{c}k+m-1 \\ m\end{array}\right)\right)^{3}\right)$ time. If $k$ is fixed, we get a polynomial time algorithm. In we choose $k=O(\log N)$, the algorithms we obtain will be "quasi-polynomial," with the complexity of $m^{O(\log N)}$.

Once a monomial expansion is obtained, it is easy to integrate polynomials since there are explicit formulas to integrate monomials. Given a monomial $\mathbf{x}^{\alpha}=$ $x_{1}^{\alpha_{1}} \cdots x_{n}^{\alpha_{n}}$, the formula is

$$
\int_{\mathbb{R}^{n}} \mathbf{x}^{\alpha} d \mu_{n}= \begin{cases}\pi^{-n / 2} \prod_{i=1}^{n} 2^{\alpha_{i} / 2} \Gamma\left(\frac{\alpha_{i}+1}{2}\right) & \text { if all } \alpha_{i} \text { are even, } \\ 0 & \text { otherwise. }\end{cases}
$$

The paper is structured as follows. In Section 2 we introduce one of the key ingredients, the Wick formula. As an application of Theorem 1.4, we describe an algorithm for approximating the hafnian of a positive matrix. In Section 3 we prove Theorem 1.4 and Corollary 1.5. In Section 4 we consider the problem of integrating polynomials with respect to the complex Gaussian measure in $\mathbb{C}^{n}$. We prove a version of Theorem 1.4 in this case and show connections between efficient complex integration and certain hard problems of combinatorial enumeration.

\section{Hafnians and the Wick Formula}

One major ingredient of the proof of Theorem 1.4 is the formula for the integral of a product of linear forms.

(2.1) Definitions. Let $m=2 k$ be an even positive integer. A perfect matching $I$ of the set $\{1, \ldots, m\}$ is an unordered partition of $\{1, \ldots, m\}$ into a union of $k$ unordered pairwise disjoint pairs

$$
I=\left\{\left\{i_{1}, j_{1}\right\},\left\{i_{2}, j_{2}\right\}, \ldots,\left\{i_{k}, j_{k}\right\}\right\} .
$$

Let $C=\left(c_{i j}\right)$ be an $m \times m$ matrix, where $m=2 k$ is an even integer. The hafnian haf $A$ of $A$ is defined by the formula

$$
\text { haf } C=\sum_{I} c_{I},
$$


where the sum is taken over all perfect matchings $I$ of the set $\{1, \ldots, m\}$ and $c_{I}$ is the product of all $c_{i j}$ for all pairs $\{i, j\} \in I$.

The following result is known as the (real) Wick formula, see, for example, [14]. Also, our proof of the complex Wick formula in Section 4 is readily modified for the real case, and, in fact, was modeled after the real case.

(2.2) Lemma. Let $m$ be a positive even integer and let $\ell_{i}: \mathbb{R}^{n} \rightarrow \mathbb{R}, i=$ $1, \ldots, m$, be linear functions. Let $C=\left(c_{i j}\right)$ be an $m \times m$ matrix defined by

$$
c_{i j}=\int_{\mathbb{R}^{n}} \ell_{i}(x) \ell_{j}(x) d \mu_{n} .
$$

Then

$$
\int_{\mathbb{R}^{n}} \prod_{i=1}^{m} \ell_{i}(x) d \mu_{n}=\operatorname{haf} C .
$$

If $\ell_{i}$ is defined by $\ell_{i}(x)=\left\langle a_{i}, x\right\rangle$ for some $a_{i} \in \mathbb{R}^{n}$, then $c_{i j}=\left\langle a_{i}, a_{j}\right\rangle$.

The problem of computing (approximating) the hafnian of a given positive matrix is of interest in combinatorics and statistical physics and generalizes the problem of computing the permanent, see Section 8.2 of [10]. Unlike in the case of the permanent, where a polynomial time approximation algorithm has recently been obtained [7], much less is known about computing hafnians.

(2.3) Computing the Hafnian of a Positive Matrix. Let $C=\left(c_{i j}\right)$ be an $m \times m$ positive symmetric matrix, where $m=2 k$ is even.

Suppose that $C$ is positive semidefinite. Then $C$ is the Gram matrix of a set of vectors, so $c_{i j}=\left\langle c_{i}, c_{j}\right\rangle$ for some vectors $c_{1}, \ldots, c_{m} \in \mathbb{R}^{m}$ and such a representation can be computed efficiently (in polynomial time). Using the Wick formula (Lemma 2.2), we can write

$$
\operatorname{haf} C=\int_{\mathbb{R}^{m}} \prod_{i=1}^{m}\left\langle c_{i}, x\right\rangle d \mu_{m} .
$$

Suppose that for each pair $c_{i}, c_{j}$ of vectors the cosine of the angle between $c_{i}$ and $c_{j}$ is at least $\delta$, which means that $c_{i j} \geq \delta \sqrt{c_{i i} c_{j j}}$ for every pair $i, j$. Then, by Theorem 1.4, to approximate haf $C$ within a factor of $(1-\varepsilon)^{m / 2}$, we can replace the integral by the integral over a random $k$-dimensional subspace $L \subset \mathbb{R}^{m}$ with $k=O\left(\varepsilon^{-2} \delta^{-2} \ln (m+2)\right)$. If $\varepsilon$ and $\delta$ are fixed in advance, we get a quasi-polynomial algorithm of $m^{O(\ln m)}$ complexity.

One can extend the above argument as follows. We observe that haf $C$ does not depend at all on the diagonal entries of $C$, so we are free to change the diagonal entries of $C$ to ensure that the above conditions are satisfied. If we put sufficiently large numbers on the diagonal of $C$, we can make sure that $C$ is positive definite, 
so $c_{i j}=\left\langle c_{i}, c_{j}\right\rangle$ for some vectors $c_{1}, \ldots, c_{m} \in \mathbb{R}^{m}$. The goal is to make the cosine of the angle between every pair $c_{i}, c_{j}$ of vectors as large as possible. Suppose that $c_{i i}=0$ for all $i$ and let $-\lambda$ be the minimum eigenvalue of $C$. Then $C+\lambda I$ is a positive semidefinite matrix and the cosine of the angle between $c_{i}$ and $c_{j}$ is $c_{i j} / \lambda$. Thus as long as the absolute value $\lambda$ of negative eigenvalues of $C$ is sufficiently small, we get an efficient algorithm to approximate haf $C$.

\section{Proofs}

Apart from the Wick formula, we need a version of the Johnson-Lindenstrauss "flattening" lemma, see, for example, [13]. We present such a version below (with nonoptimal constants), taken from Section V.7 of [1].

(3.1) Lemma. Let $x \in \mathbb{R}^{n}$ be a vector and let $L \subset \mathbb{R}^{n}$ be a k-dimensional subspace chosen at random with respect to the Haar probability measure on the Grassmannian $G_{k}\left(\mathbb{R}^{n}\right)$. Let $x^{\prime}$ be the orthogonal projection of $x$ onto L. Then, for any $0<\varepsilon<1$, the probability that

$$
(1-\varepsilon)\|x\| \leq \sqrt{\frac{n}{k}}\left\|x^{\prime}\right\| \leq(1-\varepsilon)^{-1}\|x\|
$$

is at least $1-4 \exp \left\{-\varepsilon^{2} k / 4\right\}$.

Note that the expected value of $\left\|x^{\prime}\right\|^{2} /\|x\|^{2}$ is $k / n$, so Lemma 3.1 asserts that the ratio $\left\|x^{\prime}\right\|^{2} /\|x\|^{2}$ sharply concentrates around its expectation as $k$ grows. One way to see that $\mathbf{E}\left(\left\|x^{\prime}\right\|^{2} /\|x\|^{2}\right)=k / n$ is to notice that we don't change the expected value if, instead of fixing $x$ and choosing a random $L$, we fix $L$ (e.g., to the coordinate subspace of the first $k$ coordinates) and choose a random $x \in$ $\mathbb{S}^{n-1}, x=\left(\xi_{1}, \ldots, \xi_{n}\right)$. In this case $\mathbf{E}\left(\left\|x^{\prime}\right\|^{2} /\|x\|^{2}\right)=\mathbf{E}\left\|x^{\prime}\right\|^{2}=\mathbf{E}\left(\xi_{1}^{2}+\cdots+\xi_{k}^{2}\right)$. By symmetry, $\mathbf{E} \xi_{1}^{2}=\cdots=\mathbf{E} \xi_{n}^{2}$ and since $\xi_{1}^{2}+\cdots+\xi_{n}^{2}=1$, we have $\mathbf{E} \xi_{i}^{2}=1 / n$.

The following is a straightforward corollary of Lemma 3.1. We establish it in a slightly larger generality than immediately needed, having in mind applications to complex integration in Section 4.

(3.2) Lemma. Let us choose $\delta>0$ and $\varepsilon>0$. Suppose that $a_{1}, \ldots, a_{N}$ and $b_{1}, \ldots, b_{N}$ are vectors from $\mathbb{R}^{n}$ such that the cosine of the angle between every pair $a_{i}$ and $b_{j}$ of vectors is at least $\delta>0$.

Let us choose a $\rho>0$ such that

$$
(1-\rho)^{-2} \leq 1+\frac{\delta \varepsilon}{3}
$$

and an integer

$$
k \geq \min \left\{n, 4 \rho^{-2} \ln \left(12 N^{2}+24 N\right)\right\} .
$$


Let $L \subset \mathbb{R}^{n}$ be a $k$-dimensional subspace chosen at random with respect to the Haar probability measure on the Grassmannian $G_{k}\left(\mathbb{R}^{n}\right)$. Let $a_{i}^{\prime}$, $b_{j}^{\prime}$ be the orthogonal projection of $a_{i}, b_{j}$ onto $L$. Then, with probability at least $2 / 3$,

$$
(1-\varepsilon)\left\langle a_{i}, b_{j}\right\rangle \leq \frac{n}{k}\left\langle a_{i}^{\prime}, b_{j}^{\prime}\right\rangle \leq(1-\varepsilon)^{-1}\left\langle a_{i}, b_{j}\right\rangle
$$

for all pairs $(i, j)$.

Proof. Scaling, if necessary, we may assume that $\left\|a_{i}\right\|=\left\|b_{j}\right\|=1$ for all $i$ and $j$, so $\left\langle a_{i}, b_{j}\right\rangle \geq \delta$ for all $i, j$. We have

$$
\left\langle a_{i}, b_{j}\right\rangle=\frac{\left\|a_{i}+b_{j}\right\|^{2}-\left\|a_{i}\right\|^{2}-\left\|b_{j}\right\|^{2}}{2}
$$

and

$$
\left\langle a_{i}^{\prime}, b_{j}^{\prime}\right\rangle=\frac{\left\|a_{i}^{\prime}+b_{j}^{\prime}\right\|^{2}-\left\|a_{i}^{\prime}\right\|^{2}-\left\|b_{j}^{\prime}\right\|^{2}}{2} .
$$

We note that

$$
(1-\rho)^{-2} \leq 1+\frac{\delta \varepsilon}{3} \quad \text { and } \quad(1-\rho)^{2} \geq 1-\frac{\delta \varepsilon}{3} .
$$

Since there are altogether $N^{2}+2 N$ vectors $a_{i}, b_{j}$, and $a_{i}+b_{j}$, by Lemma 3.1 , for a random $k$-dimensional subspace $L$, with probability at least $2 / 3$, we get

$$
\left\|a_{i}+b_{j}\right\|^{2}(1-\rho)^{2} \leq \frac{n}{k}\left\|a_{i}^{\prime}+b_{j}^{\prime}\right\|^{2} \leq(1-\rho)^{-2}\left\|a_{i}+b_{j}\right\|^{2}
$$

and, similarly,

$$
\left\|a_{i}\right\|^{2}(1-\rho)^{2} \leq \frac{n}{k}\left\|a_{i}^{\prime}\right\|^{2} \leq(1-\rho)^{-2}\left\|a_{i}\right\|^{2}
$$

and

$$
\mid b_{i}\left\|^{2}(1-\rho)^{2} \leq \frac{n}{k}\right\| b_{i}^{\prime}\left\|^{2} \leq(1-\rho)^{-2}\right\| b_{i} \|^{2}
$$

for all pairs $i, j$. Since $\left\|a_{i}\right\|=\left\|b_{j}\right\|=1$ and $\left\|a_{i}+b_{j}\right\| \leq 2$, we get

$$
\left\|a_{i}+b_{j}\right\|^{2}-\frac{4 \delta \varepsilon}{3} \leq \frac{n}{k}\left\|a_{i}^{\prime}+b_{j}^{\prime}\right\|^{2} \leq\left\|a_{i}+b_{j}\right\|^{2}+\frac{4 \delta \varepsilon}{3}
$$

and, similarly,

and

$$
\left\|a_{i}\right\|^{2}-\frac{\delta \varepsilon}{3} \leq \frac{n}{k}\left\|a_{i}^{\prime}\right\|^{2} \leq\left\|a_{i}\right\|^{2}+\frac{\delta \varepsilon}{3}
$$

$$
\mid b_{i}\left\|^{2}-\frac{\delta \varepsilon}{3} \leq \frac{n}{k}\right\| b_{i}^{\prime}\left\|^{2} \leq\right\| b_{i} \|^{2}+\frac{\delta \varepsilon}{3} .
$$

Therefore,

$$
\left\langle a_{i}, b_{j}\right\rangle-\delta \varepsilon \leq \frac{n}{k}\left\langle a_{i}^{\prime}, b_{j}^{\prime}\right\rangle \leq\left\langle a_{i}, b_{j}\right\rangle+\delta \varepsilon .
$$

Since $\left\langle a_{i}, b_{j}\right\rangle \geq \delta$, the proof follows. 
OF10

(3.3) Corollary. There exists an absolute constant $\gamma>0$ with the following property.

Let $\delta>0$ and $\varepsilon>0$ be numbers, let $N$ be a positive integer, and let $a_{1}, \ldots, a_{N}$ and $b_{1}, \ldots, b_{N}$ be vectors from $\mathbb{R}^{n}$ such that the cosine of the angle between every pair $a_{i}, b_{j}$ of vectors is at least $\delta$. Let $k$ be a positive integer such that

$$
k \geq \gamma \delta^{-2} \varepsilon^{-2} \ln (N+2)
$$

and let $L \subset \mathbb{R}^{n}$ be a $k$-dimensional subspace chosen at random with respect to the Haar probability measure in the Grassmannian $G_{k}\left(\mathbb{R}^{n}\right)$. Let $a_{i}^{\prime}, b_{j}^{\prime}$ be the orthogonal projections of $a_{i}, b_{j}$ onto L. Then, with probability at least $2 / 3$, we have

$$
(1-\varepsilon)\left\langle a_{i}^{\prime}, b_{j}^{\prime}\right\rangle \leq \frac{k}{n}\left\langle a_{i}, b_{j}\right\rangle \leq(1-\varepsilon)^{-1}\left\langle a_{i}^{\prime}, b_{j}^{\prime}\right\rangle
$$

for all pairs $a_{i}, b_{j}$.

The proof follows by Lemma 3.2.

Now we are ready to prove Theorem 1.4.

Proof of Theorem 1.4. We can write

$$
f(x)=\sum_{I} \alpha_{I} \prod_{i \in I}\left\langle c_{i}, x\right\rangle,
$$

where the cosine of the angle between every pair of vectors $c_{i}$ and $c_{j}$ is at least $\delta$, $I$ ranges over subsets $I \subset\{1, \ldots, N\}$ of cardinality $m$, and $\alpha_{I} \geq 0$. For every $I$, let us consider the $m \times m$ matrix $C_{I}$ whose entries $c_{i j}$ are defined by $c_{i j}=\left\langle c_{i}, c_{j}\right\rangle$. Then, by Lemma 2.2,

$$
\int_{\mathbb{R}^{n}} f d \mu_{n}=\sum_{I} \alpha_{I} \operatorname{haf} C_{I} .
$$

Let $L \subset \mathbb{R}^{n}$ be a $k$-dimensional subspace. Then the restriction $f_{L}$ of $f$ onto $L$ can be written as

$$
f_{L}(x)=\sum_{I} \alpha_{I} \prod_{i \in I}\left\langle c_{i}^{\prime}, x\right\rangle,
$$

where $c_{i}^{\prime}$ are the orthogonal projections of $c_{i}$ onto $L$. Therefore,

$$
\int_{L} f d \mu_{k}=\sum_{I} \alpha_{I} \operatorname{haf} C_{I}^{\prime},
$$

where the entries $c_{i j}^{\prime}$ of $C_{I}^{\prime}$ are defined by $c_{i j}^{\prime}=\left\langle c_{i}^{\prime}, c_{j}^{\prime}\right\rangle$. Since the hafnian of an $m \times m$ matrix is a nonnegative homogeneous polynomial of degree $m / 2$ in the entries of the matrix, the proof follows by Corollary 3.3 where we take $a_{i}=b_{i}=c_{i}$. 
Proof of Corollary 1.5. First, we claim that

$$
\max _{x \in \mathbb{S}^{n-1}} f(x)=\max _{x \in \mathbb{S}^{n-1}}|f(x)| .
$$

If the degree $m$ of $f$ is odd, this is immediate. If $m$ is even, let us consider the polynomial $f^{p}$ for some odd $p$. Since

$$
f(x)=\sum_{I} \alpha_{I} \prod_{i \in I}\left\langle c_{i}, x\right\rangle \quad \text { where } \quad \alpha_{I} \geq 0,
$$

the polynomial $f^{p}$ is also represented as a nonnegative linear combination of products of $\left\langle c_{i}, x\right\rangle$, where the cosine of the angle between every pair $c_{i}, c_{j}$ of vectors is at least $\delta$. It follows from the proof of Theorem 1.4 above that

$$
\int_{\mathbb{S}^{n-1}} f^{p} d \omega_{n}>0 \quad \text { for any } p .
$$

from which we conclude that the maximum value of $f$ and the maximum absolute value of $f$ on the sphere $\mathbb{S}^{n-1}$ must coincide.

Next, as in the proof of Theorem 1.4, we observe that if $L \subset \mathbb{R}^{n}$ is a $k$ dimensional subspace, such that for the orthogonal projections $c_{1}^{\prime}, \ldots, c_{N}^{\prime}$ of $c_{1}, \ldots, c_{N}$ onto $L$, we have

$$
(1-\varepsilon)\left\langle c_{i}^{\prime}, c_{j}^{\prime}\right\rangle \leq \frac{k}{n}\left\langle c_{i}, c_{j}\right\rangle \leq(1-\varepsilon)^{-1}\left\langle c_{i}^{\prime}, c_{j}^{\prime}\right\rangle \quad \text { for all pairs } i, j .
$$

Then

$$
(1-\varepsilon)^{m p / 2} \int_{L} f^{p} d \mu_{k} \leq\left(\frac{k}{n}\right)^{m p / 2} \int_{\mathbb{R}^{n}} f^{p} d \mu_{n} \leq(1-\varepsilon)^{-m p / 2} \int_{L} f^{p} d \mu_{k}
$$

for all $p$. In particular, if the degree $m$ of $f$ is even,

$$
\int_{\mathbb{S}^{n-1} \cap L} f^{p} d \omega_{k}>0 \quad \text { for all } p .
$$

Therefore,

$$
\max _{x \in \mathbb{S}^{n-1} \cap L} f(x)=\max _{x \in \mathbb{S}^{n-1} \cap L}|f(x)| .
$$

The proof now follows from the identities

$$
\begin{gathered}
\lim _{p \rightarrow+\infty}\left(\int_{\mathbb{S}^{n-1}} f^{2 p} d \omega_{n}\right)^{1 / 2 p}=\max _{x \in \mathbb{S}^{n-1}}|f(x)|=\max _{x \in \mathbb{S}^{n-1}} f(x), \\
\lim _{p \rightarrow+\infty}\left(\int_{\mathbb{S}^{n-1} \cap L} f^{2 p} d \omega_{k}\right)^{1 / 2 p}=\max _{x \in \mathbb{S}^{n-1} \cap L}|f(x)|=\max _{x \in \mathbb{S}^{n-1} \cap L} f(x), \\
\int_{\mathbb{S}^{n-1}} f^{2 p} d \omega_{n}=\frac{\Gamma(n / 2)}{2^{m p} \Gamma(n / 2+m p)} \int_{\mathbb{R}^{n}} f^{2 p} d \mu_{n},
\end{gathered}
$$

and

$$
\int_{\mathbb{S}^{n-1} \cap L} f^{2 p} d \omega_{k}=\frac{\Gamma(k / 2)}{2^{m p} \Gamma(k / 2+m p)} \int_{L} f^{2 p} d \mu_{k} .
$$


OF12

A. Barvinok

\section{Complex Integration}

Let $f, g: \mathbb{R}^{n} \rightarrow \mathbb{R}$ be real $n$-variate homogeneous polynomials. Let us identify $\mathbb{R}^{n} \oplus \mathbb{R}^{n}=\mathbb{C}^{n}$ via $x+i y=z$ and let $v_{n}$ be the Gaussian measure on $\mathbb{C}^{n}$ with the density

$$
\pi^{-n} e^{-\|z\|^{2}} \quad \text { where } \quad\|z\|^{2}=\|x\|^{2}+\|y\|^{2} \quad \text { for } \quad z=x+i y .
$$

We recall that $\bar{z}=x-i y$ is the complex conjugate of $z=x+i y$.

Let us define the scalar product on the space of polynomials

$$
\langle f, g\rangle=\int_{\mathbb{C}^{n}} f(z) \overline{g(z)} d v_{n}
$$

(although we use the same notation for the standard scalar product on $\mathbb{R}^{n}$, we hope no confusion will result since the domains are drastically different). One can easily check that the monomials

$$
\mathbf{x}^{\alpha}=x_{1}^{\alpha_{1}} \ldots x_{n}^{\alpha_{n}} \text { for } \alpha=\left(\alpha_{1}, \ldots, \alpha_{n}\right), \quad \text { where } \alpha_{i} \geq 0 \text { for } i=1, \ldots, n,
$$

are orthogonal under the scalar product, though not orthonormal,

$$
\left\langle\mathbf{x}^{\alpha}, \mathbf{x}^{\beta}\right\rangle= \begin{cases}\alpha_{1} ! \ldots \alpha_{n} ! & \text { if } \alpha=\beta=\left(\alpha_{1}, \ldots, \alpha_{n}\right), \\ 0 & \text { if } \alpha \neq \beta .\end{cases}
$$

Therefore, if

$$
f=\sum_{\alpha \in F} a_{\alpha} \mathbf{x}^{\alpha} \quad \text { and } \quad g=\sum_{\alpha \in G} b_{\alpha} \mathbf{x}^{\alpha}
$$

are the monomial expansions of $f$ and $g$, we have

$$
\langle f, g\rangle=\sum_{\alpha \in F \cap G} a_{\alpha} b_{\alpha} \alpha_{1} ! \cdots \alpha_{n} !
$$

It follows from the integral representation that the scalar product is invariant under the action of the orthogonal group: If $U$ is an orthogonal transformation of $\mathbb{R}^{n}$ and polynomials $f_{1}, g_{1}$ are defined by $f_{1}(x)=f(U x)$ and $g_{1}(x)=g(U x)$, then $\left\langle f_{1}, g_{1}\right\rangle=\langle f, g\rangle$.

Various problems of combinatorial counting reduce to computing the scalar products of two polynomials.

(4.1) Example. Let $a_{1}, \ldots, a_{N}$ and $b$ be some nonnegative integer $n$-vectors. Let $M$ be a positive integer. We define

$$
f(x)=\prod_{i=1}^{N}\left(\sum_{k=0}^{M} \mathbf{x}^{k a_{i}}\right) \quad \text { and } \quad g(x)=\mathbf{x}^{b} .
$$


Then the monomial expansion of $f$ contains all monomials $\mathbf{x}^{a}$, where $a$ is a linear combination of $a_{1}, \ldots, a_{N}$ with positive integer coefficients not exceeding $M$. Furthermore, if $b=\left(\beta_{1}, \ldots, \beta_{n}\right)$, then $\langle f, g\rangle$ is the number of nonnegative integer solutions $\left(k_{1}, \ldots, k_{N}\right), 0 \leq k_{i} \leq M$, to the equation

$$
k_{1} a_{1}+\cdots+k_{N} a_{N}=b
$$

times $\beta_{1}$ ! $\ldots \beta_{n}$ !. The number of such solutions $\left(k_{1}, \ldots, k_{N}\right)$ as a function of $b$ is often called the vector partition function, see [4]. Computing the vector partition function is generally as hard as counting integer points in a polytope.

(4.2) Definition. Let us fix a number $0<\delta \leq 1$ and a positive integer $N$. We say that a pair of homogeneous polynomials $f, g: \mathbb{R}^{n} \rightarrow \mathbb{R}$ of degree $m$ is $(\delta, N)$ focused if there exist $N$ nonzero vectors $a_{1}, \ldots, a_{N} \in \mathbb{R}^{n}$ and $N$ nonzero vectors $b_{1}, \ldots, b_{N} \in \mathbb{R}^{n}$ such that:

- for every pair $(i, j)$ the cosine of the angle between $a_{i}$ and $b_{j}$ is at least $\delta$;

- the polynomial $f$ can be written as a nonnegative linear combination

$$
f(x)=\sum_{I} \alpha_{I} \prod_{i \in I}\left\langle a_{i}, x\right\rangle,
$$

while the polynomial $g$ can be written as a nonnegative linear combination

$$
g(x)=\sum_{I} \beta_{I} \prod_{j \in J}\left\langle b_{j}, x\right\rangle,
$$

where the sum is taken over subsets $I, J \subset\{1, \ldots, m\}$ of cardinality $|I|=$ $|J|=m$ and $\alpha_{I}, \beta_{J} \geq 0$.

We prove that the value of the scalar product of a well-focused pair of polynomials can be well approximated from the scalar product of the restriction of the polynomials onto a random lower-dimensional subspace.

For a $k$-dimensional subspace $L \subset \mathbb{R}^{n}$, let us consider its complexification $L_{\mathbb{C}}=L \oplus i L \subset \mathbb{C}^{n}$. Let $v_{k}$ be the Gaussian measure in $L_{\mathbb{C}}$ with the density $\pi^{-k} \exp \left\{-\|z\|^{2}\right\}$ for $z \in L_{\mathbb{C}}$. We pick a $k$-dimensional subspace $L \subset \mathbb{R}^{n}$ at random with respect to the Haar probability measure on the Grassmannian $G_{k}\left(\mathbb{R}^{n}\right)$ and consider the restrictions $f_{L}$ and $g_{L}$ onto $L$ and the integral

$$
\left\langle f_{L}, g_{L}\right\rangle=\int_{L_{\mathbb{C}}} f(z) \overline{g(z)} d v_{k}
$$

We claim that as long as $k \sim \log N$, the properly scaled integral over $L_{\mathbb{C}}$ approximates the integral over $\mathbb{C}^{n}$ within a factor of $(1-\varepsilon)^{m}$.

(4.3) Theorem. There exists an absolute constant $\gamma>0$ with the following property. 
For every $\delta>0$, for any positive integer $N$, for any $(\delta, N)$-focused pair of polynomials $f, g: \mathbb{R}^{n} \rightarrow \mathbb{R}$ of degree $m$, for any $\varepsilon>0$, and any positive integer $k \geq \gamma \varepsilon^{-2} \delta^{-2} \ln (N+2)$, the inequality

$$
(1-\varepsilon)^{m}\left\langle f_{L}, g_{L}\right\rangle \leq\left(\frac{k}{n}\right)^{m}\langle f, g\rangle \leq(1-\varepsilon)^{-m}\left\langle f_{L}, g_{L}\right\rangle
$$

holds with probability at least $2 / 3$ for a random $k$-dimensional subspace $L \subset \mathbb{R}^{n}$.

The proof is very similar to that of Theorem 1.4. The only difference is that we need the complex version of the Wick formula.

(4.4) Definitions. Let $m$ be a positive integer. A permutation of the set $\{1, \ldots, m\}$ is a bijection $\sigma:\{1, \ldots, m\} \rightarrow\{1, \ldots, m\}$.

Let $C=\left(c_{i j}\right)$ be an $m \times m$ matrix. The permanent per $C$ of $C$ is defined by the formula

$$
\operatorname{per} C=\sum_{\sigma} \prod_{i=1}^{m} c_{i \sigma(i)}
$$

where the sum is taken over all permutations of the set $\{1, \ldots, m\}$.

A complex version of the Wick formula is known, see, for example, [6]. We state it below in the form we need and also provide a proof, which is a modification of the proof of the real Wick formula from [14].

(4.5) Lemma. Let $m$ be a positive integer and let $f_{i}, g_{i}: \mathbb{R}^{n} \rightarrow \mathbb{R}$ be linear functions. Let $C=\left(c_{i j}\right)$ be an $m \times m$ matrix defined by

$$
c_{i j}=\int_{\mathbb{C}^{n}} f_{i}(z) \overline{g_{j}(z)} d v_{n} .
$$

Then

$$
\int_{\mathbb{C}^{n}} \prod_{i=1}^{n} f_{i}(z) \overline{g_{i}(z)} d v_{n}=\operatorname{per} C .
$$

If $f_{i}$ is defined by $f_{i}(x)=\left\langle a_{i}, x\right\rangle$ and $g_{j}$ is defined by $g_{i}(x)=\left\langle b_{j}, x\right\rangle$ for some $a_{i}, b_{j} \in \mathbb{R}^{n}$, then $c_{i j}=\left\langle a_{i}, b_{j}\right\rangle$.

Proof. Given vectors $a_{1}, \ldots, a_{m}$ and $b_{1}, \ldots, b_{m}$, let

$$
p(x)=\prod_{i=1}^{m}\left\langle a_{i}, x\right\rangle \quad \text { and } \quad q(x)=\prod_{j=1}^{m}\left\langle b_{j}, x\right\rangle .
$$

Our goal is to prove that

$$
\langle p, q\rangle=\operatorname{per} C \quad \text { where } \quad c_{i j}=\left\langle a_{i}, b_{j}\right\rangle .
$$


First, we check the identity in the special case when $a_{1}=\cdots=a_{m}=e_{1}$, the first basis vector, and $b_{1}=\cdots=b_{m}=b=\left(\beta_{1}, \ldots, \beta_{n}\right)$ is an arbitrary vector. In this case, $p(x)=x_{1}^{m}$ and $q(x)=\left(\beta_{1} x_{1}+\cdots+\beta_{n} x_{n}\right)^{m}$, so we have $\langle p, q\rangle=\beta_{1}^{m} m$ !. On the other hand, $c_{i j}=\beta_{1}$ for all $i$ and $j$, so per $C=m ! \beta_{1}^{m}$ as well.

Next, we check the identity when $a_{1}, \ldots, a_{m}=a$ and $b_{1}, \ldots, b_{m}=b$, where $a$ and $b$ are arbitrary vectors. Applying scaling, if necessary, we can assume that $\|a\|=1$. Since an orthogonal transformation of $\mathbb{R}^{n}$ does not change either $\langle p, q\rangle$ or $C$, this case reduces to the previous one.

Now we consider the general case. We observe that both quantities $\langle p, q\rangle$ and per $C$ are multilinear and symmetric in $a_{1}, \ldots, a_{m}$ and multilinear and symmetric in $b_{1}, \ldots, b_{m}$, so we obtain the general case by polarization. For variables $\lambda=$ $\left(\lambda_{1}, \ldots, \lambda_{m}\right)$ and $\mu=\left(\mu_{1}, \ldots, \mu_{m}\right)$ we introduce vectors $a_{\lambda}=\lambda_{1} a_{1}+\cdots+\lambda_{m} a_{m}$ and $b_{\mu}=\mu_{1} b_{1}+\cdots+\mu_{m} b_{m}$. If $F\left(a_{1}, \ldots, a_{m} ; b_{1}, \ldots, b_{m}\right)$ is any polynomial multilinear and symmetric in $a_{1}, \ldots, a_{m}$ and multilinear and symmetric in $b_{1}, \ldots, b_{m}$, then $(m !)^{2} F\left(a_{1}, \ldots, a_{m} ; b_{1}, \ldots, b_{m}\right)$ is equal to the coefficient of the product $\lambda_{1} \cdots \lambda_{m} \mu_{1} \cdots \mu_{m}$ in the expansion of $F\left(a_{\lambda}, \ldots, a_{\lambda} ; b_{\mu}, \ldots, b_{\mu}\right)$ as a polynomial in $\lambda_{1}, \ldots, \lambda_{m}, \mu_{1}, \ldots, \mu_{m}$. Since if two such polynomials $F$ and $G$ agree on all (2m)-tuples $(a, \ldots, a ; b, \ldots, b)$, they agree everywhere. Letting $F=\langle p, q\rangle$ and $G=\operatorname{per} C$, we complete the proof.

Now the proof of Theorem 4.5 follows the proof of Theorem 1.4.

\section{Acknowledgment}

This research was partially supported by NSF Grant DMS 0400617.

\section{References}

[1] A. Barvinok, A Course in Convexity, Graduate Studies in Mathematics, Vol. 54, American Mathematical Society, Providence, RI, 2002.

[2] A. Barvinok, Estimating $L^{\infty}$ norms by $L^{2 k}$ norms for functions on orbits, Found. Comput. Math. 2 (2002), 393-412.

[3] B. J. C. Baxter and A. Iserles, On the Foundations of Computational Mathematics, Handbook of Numerical Analysis, Vol. XI, North-Holland, Amsterdam, 2003, pp. 3-34.

[4] M. Brion and M. Vergne, Residue formulae, vector partition functions and lattice points in rational polytopes, J. Amer. Math. Soc. 10 (1997), 797-833.

[5] L. Faybusovich, Global Optimization of Homogeneous Polynomials on the Simplex and on the Sphere, Frontiers in Global Optimization, Nonconvex Optim. Appl., Vol. 74, Kluwer Academic, Boston, MA, 2004, pp. 109-121.

[6] L. Gurvits, Classical complexity and quantum entanglement, J. Comput. System Sci. 69 (2004), 448-484.

[7] M. Jerrum, A. Sinclair, and E. Vigoda, A polynomial-time approximation algorithm for the permanent of a matrix with nonnegative entries, J. ACM 51 (2004), 671-697. 
[8] E. Kaltofen and L. Yagati, Improved sparse multivariate polynomial interpolation algorithms, Symbolic and Algebraic Computation (Rome, 1988), Lecture Notes in Comput. Sci., Vol. 358, Springer-Verlag, Berlin, 1989, pp. 467-474.

[9] E. De Klerk, M. Laurent, and P. Parrilo, On the equivalence of algebraic approaches to the minimization of forms on the simplex, Positive Polynomials in Control, Lecture Notes in Control and Inform. Sci., Vol. 312, Springer-Verlag, Berlin, 2005, pp. 121-132 .

[10] H. Minc, Permanents, Encyclopedia of Mathematics and its Applications, Vol. 6, Addison-Wesley, Reading, MA, 1978.

[11] V. D. Milman and G. Schechtman, Asymptotic Theory of Finite-Dimensional Normed Spaces, with an Appendix by M. Gromov, Lecture Notes in Mathematics, Vol. 1200, Springer-Verlag, Berlin, 1986.

[12] J. Renegar, On the computational complexity and geometry of the first-order theory of the reals, I. Introduction. Preliminaries. The geometry of semi-algebraic sets. The decision problem for the existential theory of the reals (255-299); II. The general decision problem. Preliminaries for quantifier elimination (301-327); III. On the computational complexity and geometry of the firstorder theory of the reals; III. Quantifier elimination (329-352), J. Symbolic Comput. 13 (1992), $255-352$.

[13] S. S. Vempala, The Random Projection Method, DIMACS Series in Discrete Mathematics and Theoretical Computer Science, Vol. 65, American Mathematical Society, Providence, RI, 2004.

[14] A. Zvonkin, Matrix integrals and map enumeration: An accessible introduction, Combinatorics and Physics (Marseilles, 1995), Math. Comput. Modelling 26 (1997), 281-304. 